\title{
High-Frequency Electrooptic Fabry-Perot Modulators
}

\author{
R. J. Simes, R. H. Yan, C. C. Barron, D. Derrickson, D. G. Lishan, J. Karin, L. A. Coldren, \\ M. Rodwell, S. Elliott, and B. Hughes
}

\begin{abstract}
Electrooptic modulators built from GaAs/ $\mathrm{Al}_{x} \mathrm{Ga}_{1-x}$ As Fabry-Perot cavities operating up to $6.5 \mathrm{GHz}$ are reported. The measured frequency response agrees well with the one predicted using an equivalent circuit model derived from high-speed electrical measurements. The parasitic capacitances have been reduced to $\sim \mathbf{3 0} \mathrm{fF}$ by fabricating the devices on semi-insulating GaAs substrates and integrating them with onwafer bond pads which have dimensions compatible with microwave coplanar probes.
\end{abstract}

$\mathrm{D}$ RAMATIC improvements in the dc response of surface-normal electrooptic modulators have been achieved in the last two years [1]-[4]. Reflectivity modulation of $50 \%$ is possible with drive voltages as low as $2 \mathrm{~V}$. The low drive voltage of these modulators combines with their inherently low capacitance to make them ideal for high-frequency applications [5], [6]. Boyd et al. [5] have demonstrated $5 \mathrm{GHz}$ operation of a wire-bonded, transverse modulator built on an $\mathrm{n}^{+}$substrate; in that structure, the parasitic capacitance of the device connection limited the modulation speed. In the present letter, we report the fabrication and high-frequency electrical and optical characterization of a transverse modulator on a semi-insulating substrate.

\section{Modulator Design and Fabrication}

The modulator consists of a symmetric Fabry-Perot (FP) cavity with a multiple-quantum-well (MQW) active region [7]. A cross-sectional schematic and a top-view photograph of the structure are shown in Fig. 1. The MQW (100- $\AA$ $\mathrm{GaAs} / 100-\AA \quad \mathrm{Al}_{0.2} \mathrm{Ga}_{0.8} \mathrm{As}$ ) active region is three wavelengths thick, yielding a drive voltage of $8-10 \mathrm{~V}$. A passive n-doped region, incorporated in the Fabry-Perot intracavity medium, forms the n-contact layer. The top mirror, doped $\mathrm{p}$

Manuscript received March 15, 1991. This work was supported by the U.S. Air Force Office of Scientific Research. The work of R. J. Simes was partially supported by a fellowship from the American Electronics Association.

R. J. Simes was with the Department of Electrical and Computer Engineering, University of California, Santa Barbara, CA 93106. He is now with Alcatel Alsthom Recherche, 91460 Marcoussis, France.

R. H. Yan was with the Department of Electrical and Computer Engineering, University of California, Santa Barbara, CA 93106 . He is now with AT\&T Bell Laboratories, Holmdel, NJ 07733.

C. C. Barron, D. Derrickson, D. G. Lishan, J. Karin, L. A. Coldren, and M. Rodwell are with the Department of Electrical and Computer Engineering, University of California, Santa Barbara, CA 93106.

S. Elliott and B. Hughes are with the Hewlett-Packard Company, Santa Rosa, CA 95403.

IEEE Log Number 9100950.

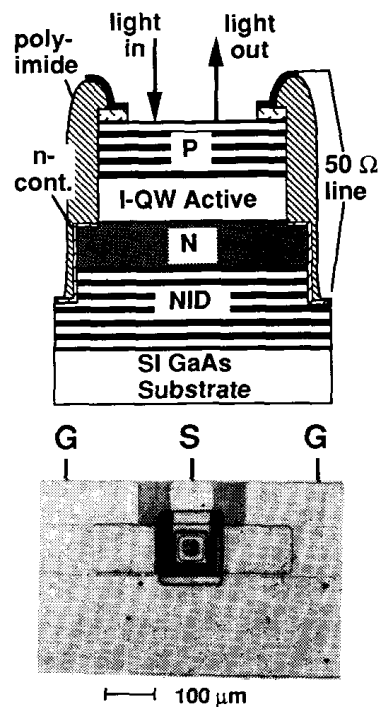

Fig. 1. Schematic representation of the modulator, and a photograph of the completed structure. The top mirror consists of nine periods of AlAs (733 $\AA) / \mathrm{Al}_{0.2} \mathrm{Ga}_{0.8} \mathrm{As}(633 \AA)$ doped $\mathrm{p}$ type, and the bottom mirror consists of 12.5 periods of undoped material. The $\mathrm{n}$-contact layer is $1.0 \mu \mathrm{m}$ of $x=0.15 \mathrm{Al}_{x} \mathrm{Ga}_{1-x} \mathrm{As}(4 \lambda / n)$, and the MQW layer is $-0.75 \mu \mathrm{m}(3 \lambda / n)$ of undoped MQW material (100- $\AA$ GaAs wells with $100-\AA x=0.2$ barriers). In the photograph, the signal line $(S)$ climbs over the polyimide to make contact to the top p contact. The ground lines $(G)$ are connected to the $n$ contact at both sides of the active region.

type to $-2 \times 10^{18} \mathrm{~cm}^{-3}$, forms the p-contact layer; its heterointerfaces were linearly graded to reduce the series resistance [8]. Modulators with three different electrically active areas were fabricated: $42 \times 42 \mu \mathrm{m}, 62 \times 62 \mu \mathrm{m}$, and $102 \times 102 \mu \mathrm{m}$. The mask set also includes bond pads with short, open, and resistive terminations for electrical calibration. The fabrication procedure involves six mask steps: 1) $\mathrm{Cr}-\mathrm{AuZn}-\mathrm{Au}$ p-ohmic ring contact formation by a liftoff process, 2) wet chemical etching $\left(1: 5: 15 \mathrm{H}_{2} \mathrm{SO}_{4}: \mathrm{H}_{2} \mathrm{O}_{2}: \mathrm{H}_{2} \mathrm{O}\right)$ through the $\mathrm{p}$ mirror and the $\mathrm{i}$-active layer to expose the n-contact layers, 3 ) wet-chemical etching through the $\mathrm{n}$ layer and bottom mirror to isolate devices, 4) $\mathrm{AuGe}-\mathrm{Ni}-\mathrm{Au} \mathrm{n}$ contact deposition, 5) patterning of the polyimide dielectric layer to prevent junction shorting, and 6) deposition of final contact metal ( $\mathrm{Ti}-\mathrm{Au})$ to form on wafer-bond pads compatible with microwave coplanar probes. The pads' width and separation are such that they form a transmission line with an intrinsic impedance of $50 \Omega$ [9]. 
TABLE I

Summary of Measured Modulator Characteristics. The 1-MHz Capacitance Value Includes the Bond-Pad Capacitance

of $30 \mathrm{fF}$, Whereas the 8510 Measurement Represents the Capacitance of the Modulator Only. For the 8510

Measurements, the $R$ and $C$ Values were Chosen at 5, 4, and $1.2 \mathrm{GHz}$ for the Small, Medium, and

Large Diodes, Respectively. The Predicted 3-dB Frequency is Given by $1 / 2 \pi C(50 \Omega+R)$ for Devices of Series Resistance $R$ and Capacitance $C$ in a 50- $\Omega$ System [5]

\begin{tabular}{cccccccc}
\hline & \multicolumn{2}{c}{$\mathrm{dc} / 1 \mathrm{MHz}$ Meas. } & \multicolumn{2}{c}{8510 Meas. } & & \\
Diode Size & $R(\Omega)$ & $C(\mathrm{fF})$ & $R(\Omega)$ & $C(\mathrm{fF})$ & Calc. $f_{3 \mathrm{~dB}}(\mathrm{GHz})$ & Meas. $f_{3 \mathrm{~dB}}(\mathrm{GHz})$ \\
\hline $42 \times 42 \mu \mathrm{m}^{2}$ & 30 & 330 & 36 & 280 & 6.6 & $6.5 \mathrm{GHz}$ \\
$62 \times 62 \mu \mathrm{m}^{2}$ & 18 & 670 & 20 & 620 & 3.3 & $3.3 \mathrm{GHz}$ \\
$102 \times 102 \mu \mathrm{m}^{2}$ & 12 & 1730 & 14 & 1710 & 1.5 & $1.0 \mathrm{GHz}$ \\
\hline
\end{tabular}

\section{Electrical Measurements and Modeling}

Table I lists the capacitance at $1 \mathrm{MHz}$ and the dc series resistance of the completed modulators. For the smallest device, we measured a capacitance at $1 \mathrm{MHz}$ of $290 \mathrm{fF}$ after mask step 4. The measured capacitance at $1 \mathrm{MHz}$ of the coplanar bond pads alone was $30 \mathrm{fF}$. The area overlap of the bond pad metal with the buried $\mathrm{n}^{+}$layer $\left(\sim 800 \mu \mathrm{m}^{2}\right.$ in area separated by $\sim 2 \mu \mathrm{m}$ of polyimide) adds $\sim 10 \mathrm{fF}$ of extra capacitance. The sum of these values agrees well with the measured value, $330 \mathrm{fF}$, of the completed device. The series resistance $R$ results from the sheet resistivity of the buried $n$ layer, measured to be $35 \Omega / \mathrm{sq}$, and the series resistance of the $\mathrm{p}$ mirror.

The modulators were characterized in the gigahertz frequency range using an HP 8510 network analyzer configured with $50-\Omega$ high-speed probes. As determined by measurements of shorted and open bond pads, the impedance of the on-wafer bond pads alone can be well modeled by a $30-\mathrm{pH}$ series inductance and a $30-\mathrm{fF}$ shunt capacitance. For a $50-\Omega$ transmission line of the same length as the bond pads, we calculate $30 \mathrm{pH}$ and $12 \mathrm{fF}$ [9]. We attribute the additional capacitance, $\sim 20 \mathrm{fF}$, to either the pad termination at the modulator or impedance variations at the probe/pad interface. The total parasitic capacitance is approximately $30 \mathrm{fF}$ : $20 \mathrm{fF}$ from the bond pads, and $10 \mathrm{fF}$ from the final metal/buried n-layer overlap.

The impedance of the modulator alone was determined by subtracting the contribution of the on-wafer pads from the impedance of the modulator/pad combination. The behavior of the modulator's impedance as a function of frequency can be modeled well by a simple series $R-C$ circuit. At a reverse bias of $10 \mathrm{~V}$, the series resistance thus determined for the smallest device varied by $\pm 16 \%$ about the value of $36 \Omega$ at $5 \mathrm{GHz}$ as the frequency was swept from 0.5 to $12 \mathrm{GHz}$. Over the same bandwidth, the capacitance of $0.28 \mathrm{pF}$ at 5 $\mathrm{GHz}$ varied by $\pm 7 \%$.

\section{Optical Measurements}

A schematic of the measurement system is shown in Fig. 2. A tunable Ti:sapphire laser is coupled to an optical microscope, making it possible to image the laser spot and the modulator together. The response of the source/spectrum analyzer and the cables was calibrated directly by connecting the output of the source to the spectrum analyzer using the

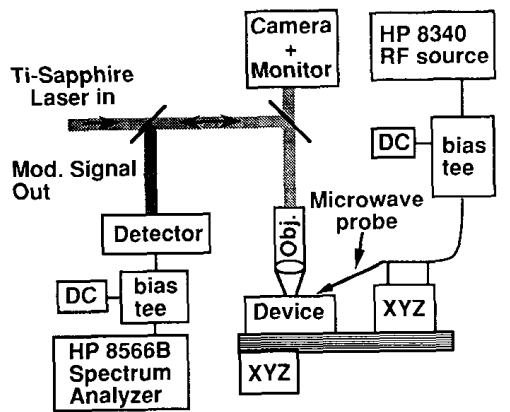

Fig. 2. Gigahertz electrooptic modulator characterization probe station The laser has been coupled in and out of the optical column of a microscope to facilitate imaging the device active area and the laser spot. The laser input had a Gaussian beam waist of $-8 \mu \mathrm{m}$, and the power incident on the modulator was $-2 \mathrm{~mW}$.

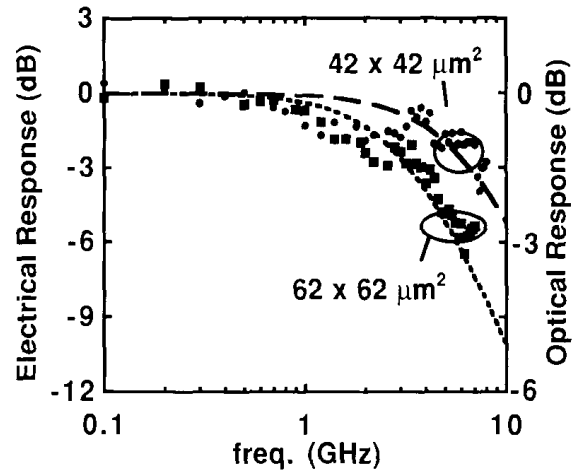

Fig. 3. Normalized electrical and optical modulation efficiency versu frequency are presented for $42 \times 42 \mu \mathrm{m}^{2}(\bullet)$ and $62 \times 62 \mu \mathrm{m}^{2}$ (V) modulators; the data are corrected for the system response. The electrical power refers to the power produced by the current output from the high-speed detector in the spectrum analyzer. The dashed line represents the corresponding calculated response based on the gigahertz-frequency-determined model parameters. The data were taken at $\sim 900 \mathrm{~nm}$, and the voltage bias voltage was $\sim 10 \mathrm{~V}$.

same cabling, and the detector response was calibrated using a GaAs wavelength picosecond pulse train laser [10].

Small-signal measurements were made by superimposing an $\mathrm{RF}$ input of $4 \mathrm{dBm}$ on a dc bias voltage of 10 . The input light wavelength was tuned to optimize the optical modulation $(\lambda \sim 900 \mathrm{~nm})$. The FP resonance wavelength for this device was further from the $\mathrm{QW}$ exciton absorption peak than intended, increasing the bias voltage requirement and accen- 
tuating the index-modulation aspect for these devices. The $\mathrm{dc}$ reflectivity modulation at the measurement bias and wavelength was $\sim 5 \% / V$. In Fig. 3, we show a normalized frequency response plot for two different sizes of modulators. We also show the expected frequency response behavior calculated using the model parameters determined at high frequency. There is a small ripple in the measured data at 2 $\mathrm{GHz}$, which we attribute to uncalibrated reflections at the transition between the coaxial cable and the coplanar waveguide in the high-speed probe. The measured frequency (meas. $f_{3 \mathrm{~dB}}$ ) and calculated frequency (calc. $f_{3 \mathrm{~dB}}$ ) for all three sizes of diodes are listed in Table I. These results are for an unterminated structure; by terminating the modulator with a $50-\Omega$ load, the effective $3-\mathrm{dB}$ bandwidth for the smallest structure could be increased to greater than $10 \mathrm{GHz}$ [11]. The present device is operating at a wavelength $30-40$ $\mathrm{nm}$ from the exciton absorption peak; thus, the index modulation is augmented, and there is little light absorption in the active region. As a result, we do expect the present modulators to be limited by the electron/hole escape times from the $M Q W$ region [12].

\section{Conclusion}

We have demonstrated gigahertz operation of a FabryPerot modulator. The parasitic capacitance of the device connection has been reduced to $\sim 30 \mathrm{fF}$ by fabricating the device on a semi-insulating substrate. The present structure was not completely optimized; hence; only small-signal measurements were made. An optimized device with the same structure would yield a figure of merit $F$, as defined by Walker [11], of approximately $1 \mathrm{GHz} \cdot \mathrm{V}^{-1} \cdot \mu \mathrm{m}$. That value is similar to those observed for waveguide structures. By exploiting the FP structure further [1], [2], devices exhibiting $5-10 \mathrm{GHz} \cdot \mathrm{V}^{-1} \cdot \mu \mathrm{m}$ should be readily realizable.

\section{ACKNOWLEDGMENT}

This work benefited from the input of D. Braun, E. Ehlers, and R. Van Tuyl of Hewlett-Packard, and J. Bowers and D. Cohen of the University of California, Santa Barbara.

\section{REFERENCES}

[1] R. H. Yan, R. J. Simes, and L. A. Coldren, "Extremely low-voltage Fabry-Perot reflection modulators," IEEE Photon. Technol. Lett., vol. 2, p. 118, 1990.

[2] M. Whitehead, A. Rivers, G. Parry, J. S. Roberts, and C. Button, "Low-voltage multiple quantum well reflection modulator with on:off ratio > 100:1," Electron. Lett., vol. 25, pp. 984-985, 1989.

[3] H. Fouckhardt, M. Walther, T. Hackbarth, and K. J. Ebeling, "Design of epitaxial AIGaAs multilayer structures and optically controllable reflection modulators," Opt. Laser Technol., vol. 22, pp. $22-30,1990$.

[4] B. Pezeshki, D. Thomas, and J. S. Harris, "Optimization of modulation ratio and insertion loss in reflective electroabsorption modulators," Appl. Phys. Lett., vol. 57, p. 1491, 1990.

[5] G. D. Boyd, J. E. Bowers, C. E. Soccolich, D. A. B. Miller, and D. S. Chelma, "5.5 GHz multiple quantum well reflection modulator," Electron. Lett., vol. 25, pp. 558-559, 1989.

[6] M. Walther and K. J. Ebeling, "Gigabit operation of an MQW modulator with integrated Fabry-Perot resonator," Proc. European Conf. Opt. Commun., 1989, vol. 1, pp. 225-229.

[7] R. H. Yan, R. J. Simes, and L. A. Coldren, "Analysis and design of surface-normal Fabry-Perot electro-optic modulators," IEEE J. Quantum Electron., vol. 25, pp. 2272-2280, 1989.

[8] R. S. Geels, S. W. Corzine, J. W. Scott, D. B. Young, and L. A. Coldren, "Low threshold planarized vertical-cavity surface-emitting lasers,"' IEEE Photon. Technol. Lett., vol. 2, p. 234, 1990.

[9] K. G. Gupta, R. Garg, and I. J. Bahl, Microstrip Lines and Slotlines. Dedham, MA: Artech House, 1979.

[10] J. E. Bowers and C. A. Burrus, "Ultrawide-band long-wavelength p-i-n photodetectors," J. Lightwave Technol., vol. LT-5, pp. $1339-1350,1987$.

[11] R. G. Walker, "Broadband (6 GHz) GaAs/AlGaAs electro-optic modulator with low drive power," Appl. Phys. Lett., vol. 54, pp. 1613-1615, 1989.

[12] G. D. Boyd, A. M. Fox, D. A. B. Miller, L. M. F. Chirovsky, L. A D'Asaro, J. M. Kuo, R. F. Kopf, and A. L. Lentine, "33 optical switching of symmetric self-electro-optic effect devices," Appl. Phys. Lett., vol. 57, pp. 1843-1845, 1990. 